\title{
THE DESIGN OF DISPLAY DIGITAL DATA INTERFACE CLAMP-METER COMPLEMENTED BY SENSOR GMR (GIANT MAGNETORESISTANCE)
}

\author{
Yulkifli*, Fitri Afriani, Yohandri, Ramli \\ ${ }^{1}$ Department of Physics, University of Padang,Jl. Prof Hamka, Air Tawar r Padang 25131, Indonesia \\ *Corresponding Author Email: yulkifliamir@gmail.com
}

Received: 28 March 2020

Revised: 26 April 2020

Accepted: 26 April 2020

Online: 27 April 2020

Published: 30 April 2020

SPEKTRA: Jurnal Fisika dan Aplikasinya

p-ISSN: 2541-3384

e-ISSN: 2541-3392

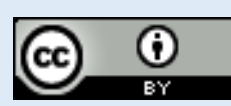

\begin{abstract}
This article discusses the design of a robust data interface system that displays an electric current using the GMR sensor. Robust measurement of electrical current to detect magnetic fields contained in the current-carrying wire. The magnetic field on the cable should be the input signal to the GMR sensor to be processed by the interface program. This interface used the Arduino IDE program and displayed in the LCD screen. This research is an experimental research laboratory. The results of the data interface can be displayed in the form of comparative measurements with the power of the conventional electric current. Comparison measurements can be viewed in graphical form. The results of the study have an average measurement accuracy of $91.2 \%$, with an average of 0.96 . Besides, this study also obtained a standard deviation of 0.21 and an average error of $0: 08$.
\end{abstract}

Keywords: GMR, clamp-meter, interface, Arduino Uno 


\section{INTRODUCTION}

In modern times, technology is developing rapidly. Advances in technology related to the advancement of physics [1], and therefore the physics become one of the best contributors to world technology. The technological advances of physics, including the interface to the measuring system. The programming interface is also experienced in the data detection magnetic field.

The interface string magnetic fields to detect electrical currents have existed, which uses the hall effect sensor. The principle works like the working principle of the magnetometer; sensors could feel the strength of the applied magnetic flux. However, unlike simple inductive sensors, hall effect sensors will work when the magnetic flux used is static. Clamp as media contain toroidal iron core with two slits contain hall effect sensor chip so that the induced magnetic flux from current-carrying wires routed through it [2]. However, this interface can only measure current strength in large numbers so strong for the measurement of electric current in a small size is very difficult to do. Therefore,

Based on the characterization, the GMR sensor can measure a magnetic field with a sensitivity of $120 \mathrm{~V} / \mathrm{T}$ with $1 \mathrm{MHz}$ response time. This sensor also has a low power consumption that is $10 \mathrm{mw}$, and miniature sensor size is $10-100 \mu \mathrm{m}$ [3].

In this study, the GMR sensor used is the type of GMR AA002-02. Sensors of this type have sensitivity high when compared with other types. GMR sensors will work if it detects the magnetic field around it [4].

GMR sensor output in the order of millivolts, so it takes a maximum signal amplifier circuit in order volts. The signal amplifier circuit that is used is the IC instrumentation amplifier AD620. AD620 IC is suitable for systems that require high precision such as scales, medical applications such as ECG and blood pressure monitoring [5]. The output signal is processed using an Arduino Uno microcontroller. Arduino microcontroller to function as an analog signal processing in getting the GMR sensor. The Arduino Uno microcontroller using C language as a programming language Arduino board [6]. Besides, Arduino Uno is open source, which can identify the neighborhood easily through the various sensors used. Arduino Uno has a processing power ATMEL328 $\mathrm{u} / \mathrm{pu}$ with the miniature package with interfacing and programming capabilities that are easy for beginners to the experts [7].

Arduino Uno is a combination of development tools and hardware, programming language, and Integrated Development Environment (IDE) that is advanced. Besides, the Arduino chip device requires no program because it has a bootloader that will handle uploading from a computer with a USB program as a means of communication. Arduino programming language is also easy because the Arduino software comes with a library collection that is quite complete. Arduino also has a ready-made module (shield) that can be connected to the Arduino board [8]. 
After the output decoded by software interfacing, then the measurement results displayed on the LCD screen. LCD functions as a medium for displaying sensor values, text value, or a menu in an application microcontroller provides many advantages for the user [9]. A microcontroller base owned by LCD allows users to display the output in a display that is energy efficient, lightweight, and relatively easy design process. Besides, LCD can also display the ASCII-based characters and can display the characters as desired [10].

\section{METHOD}

This research is an experimental research. GMR sensors are attached to the side of the clamp will detect magnetic fields on the current-carrying wire. The signals generated by the GMR sensor signal is forwarded to the amplifier circuit to be processed in the microcontroller. Signal processing results will be displayed on the LCD screen. In general, a block diagram is shown in FIGURE 1.

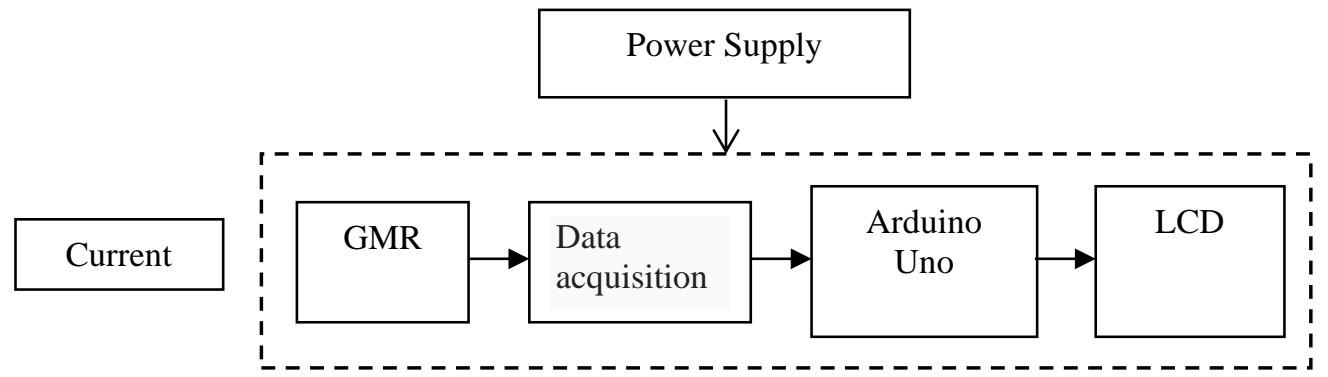

FIGURE 1. The Block Diagram Clamp-meter

The software design of this system in the form of a flowchart of a program for the microcontroller using $\mathrm{C}$ language Flowchart of Clamp meter can be seen in FIGURE 2. 


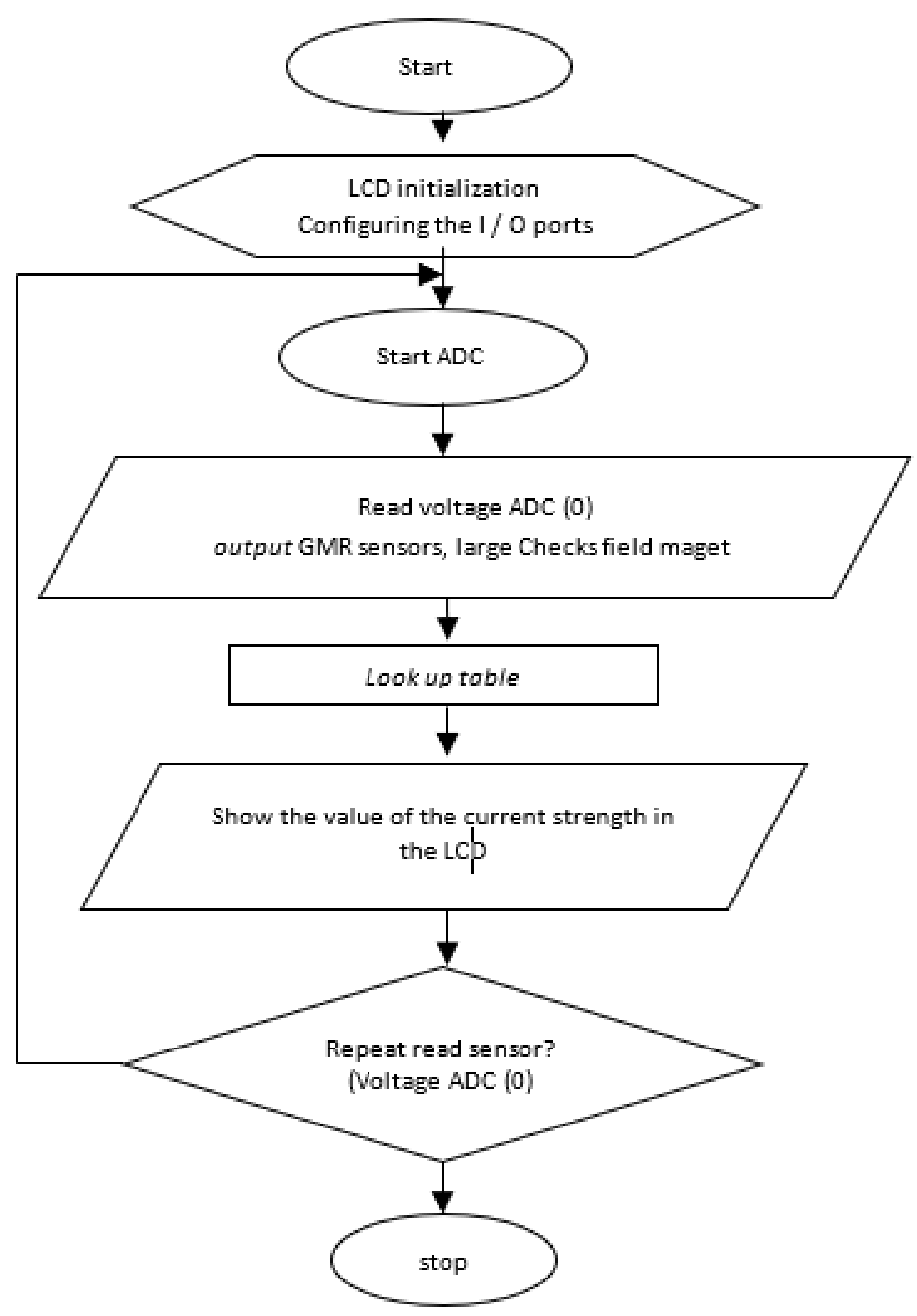

FIGURE 2. The Flowchart Program Clamp-meter System

\section{RESULT AND DISCUSSION}

In this study produced a display system analog signals into digital data to a data form strong electric current using the GMR sensor. Analysis of obtained data interface in the form of performance specifications and design of interfaces Clamp meters.

The results of the performance of the interface specification are a series of Clamp-meters (1) power supply, (2) signal amplifier circuit, (3) Microcontroller circuit, (4) LCD port, and (5) GMR sensor port as shown in FIGURE 3. 


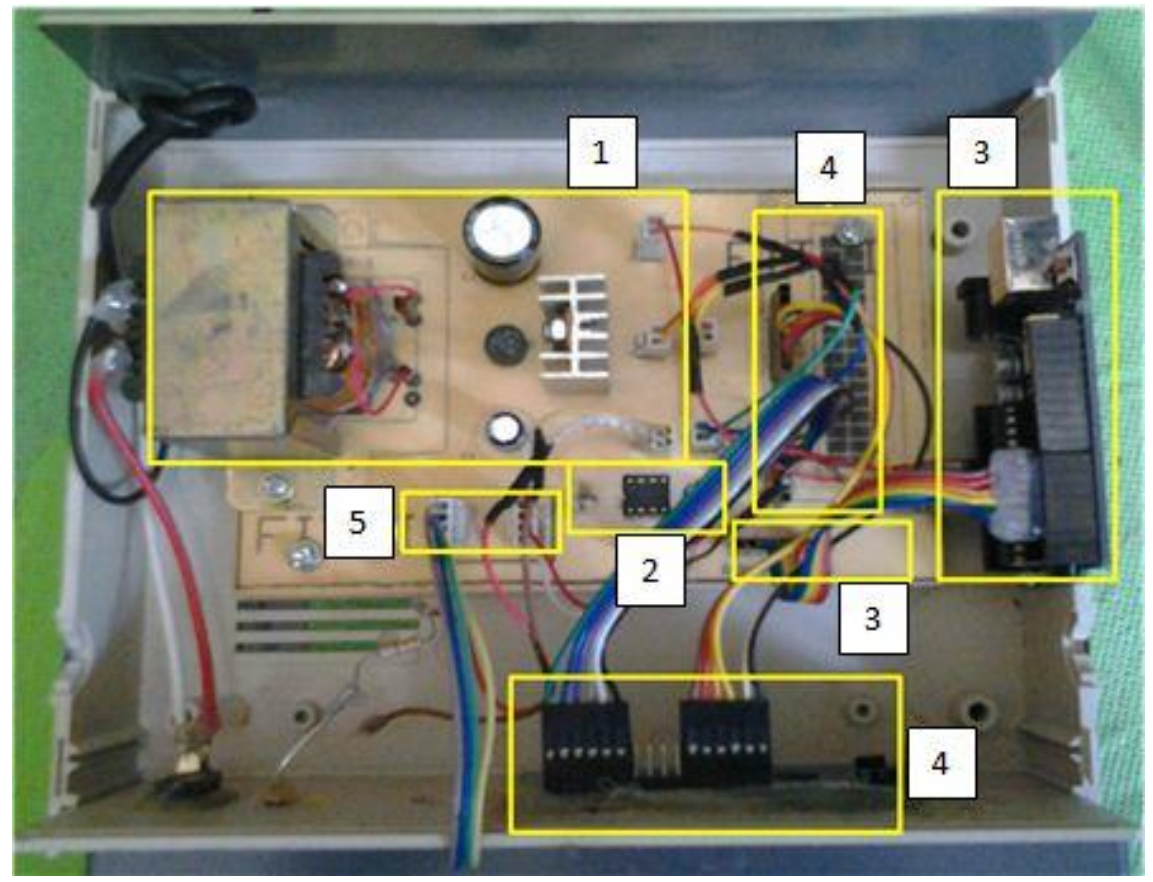

FIGURE 3. The circuit Electronic Data Processing Module

In carrying out this program, given the current strength of data input from the GMR sensor in the form of the magnetic field contained in the current-carrying wire. If the current strength is getting higher, the greater the magnetic field so that sensors detect and produce output that is proportional to the current strength in the current-carrying cable. Data received by the sensor is analog data, and will be forwarded to the microcontroller to be processed into binary data and can be displayed on display such as an LCD. Output in the LCD displayed like in FIGURE 4:

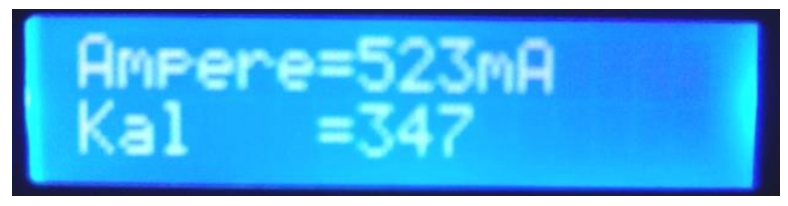

FIGURE 4. Display LCD display

To investigate the effect of changing the electrical current strength against the sensor output voltage characteristics of the sensor that is done by varying the current strength will be altered. The data obtained can be plotted graph by placing a strong current on the horizontal axis (X) and voltage sensors on the vertical axis (Y), as in FIGURE 5. 


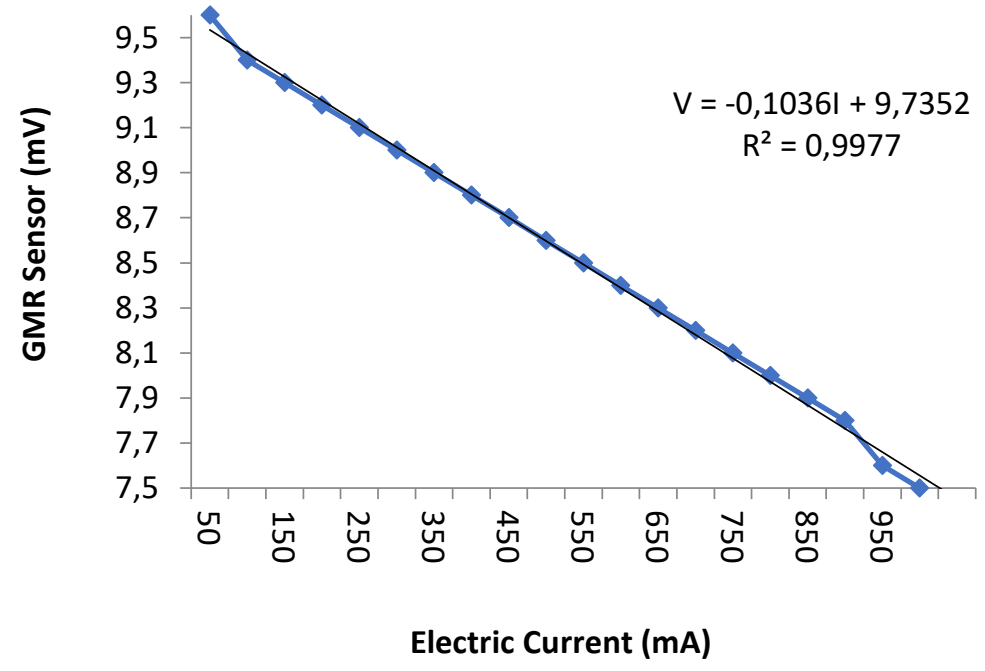

FIGURE 5. The GMR Sensor Characteristics Graph

FIGURE 5 explained that the GMR sensor output variation of the current strength is inverse, the greater, the stronger the applied current, the smaller the GMR sensor output. It is caused by a change in resistance, which is contained in the GMR sensor due to changes in the magnetic field. The list of barriers is designed integrated with a Wheatstone bridge resistive element is comprised of two active sensing elements, and the two elements are protected. So that each of the current strength in the cable is increased as much as $50 \mathrm{~mA}$, the output voltage of the sensor will respond with output worth $0.1 \mathrm{mV}$.

The design of the interface refers to the flowchart in FIGURE 2, which is processed using an IDE program Arduino Uno generate strong electrical current measurement data using Clamp meters. The measured data will be compared with measurements of the electric current strength using amperemeter. The results of the appearance of the data can be viewed in FIGURE 6.

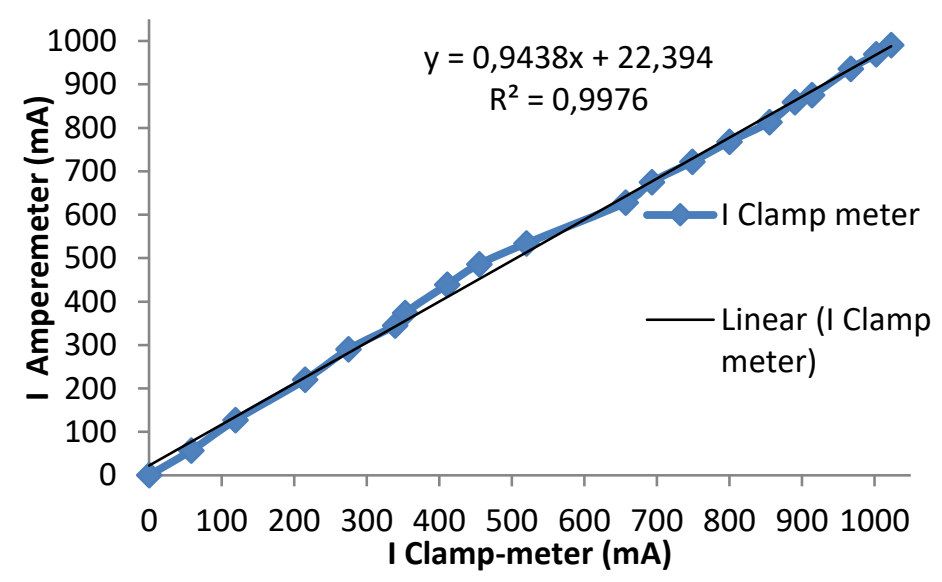

FIGURE 6. The plot strong electric current measurement results using the interface GMR.

FIGURE 6 describes the comparison measurement using Clamp meter and amperemeter. Comparisons between the two measurement results are linear with the determinant value 
reached 0.9976 in terms of the measurement of the measuring system is almost close to the actual measurement values.

Meanwhile, the sensitivity of the measurement system can be seen from the strong influence of the voltage source current is generated both on amperemeter and Clamp meters. In this measurement, the sensitivity seen from the comparison between the two measurements are measurements on amperemeter and Clamp meter with the same current source. This comparison can be seen in FIGURE 7.

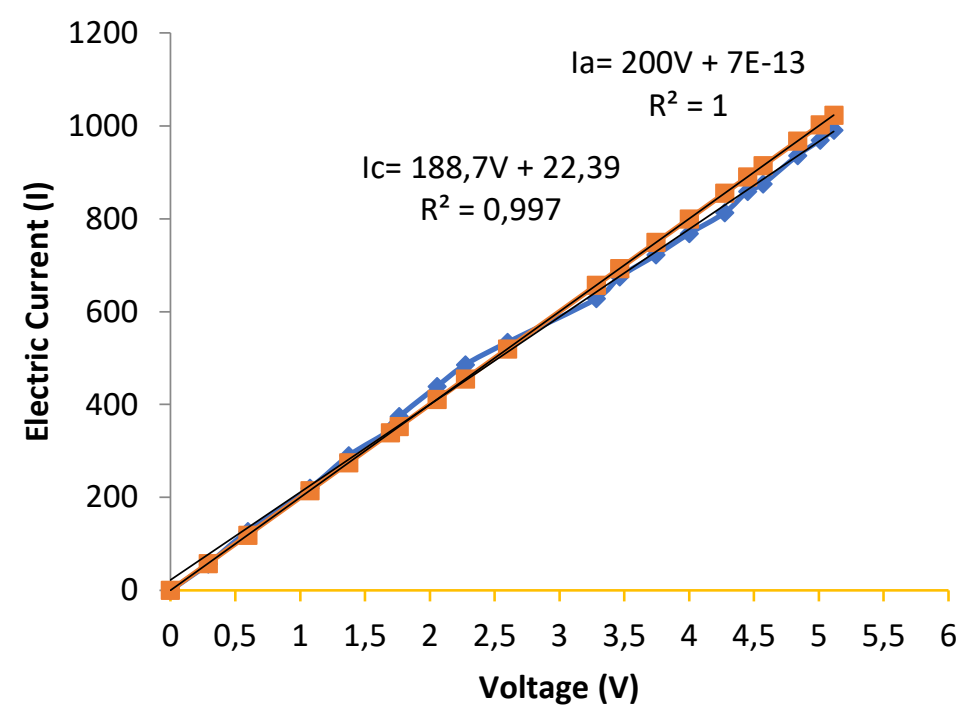

FIGURE 7. The Sensitivity Measure Tool Clamp-meter

To test the accuracy system, it would require repeated measurements. Measurement of currentcarrying wire current strength at each performed ten times measurement. They can be determined based on the average value, standard deviation, error percentage, and accuracy.

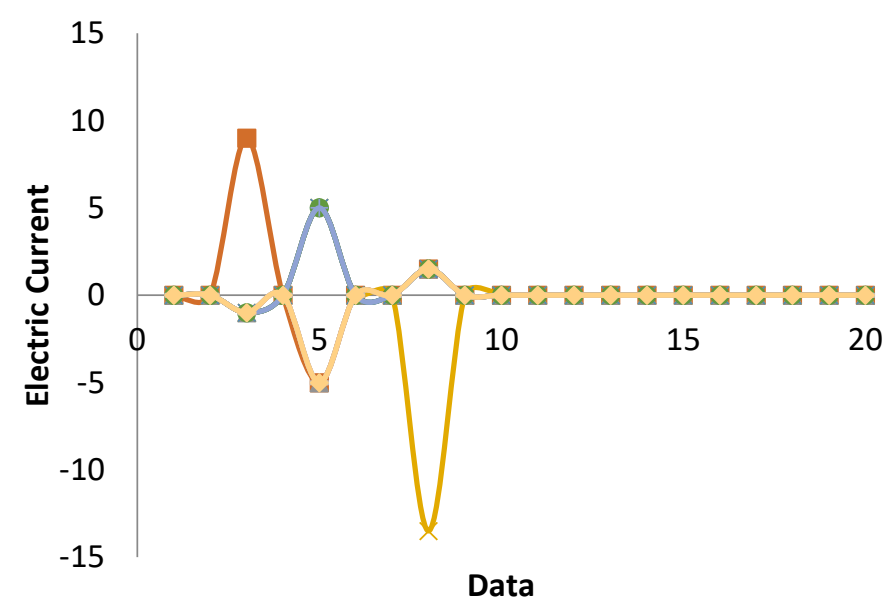

FIGURE 8. The Graph Accuracy Measurement System

FIGURE 8 explains that the accuracy of the measurement system can be seen from the standard deviation of the repeated measurements. The measurement system has an accuracy 
that is from 0.93 to 1 , or the average accuracy is 0.96 , with a standard deviation of the average is 0.21 . The average error percent, reaching $0.08 \%$. These error show that these tools have high precision.

\section{CONCLUSION}

A digital display design interface data clamp meter has been done. This study uses a GMR sensor AA002-02 types of data obtained is accurate enough for a high-sensitivity sensor. This design consists of hardware that GMR sensor, Power supply, signal amplifier circuit, microcontroller, and LCD. Data generated by the GMR sensor is processed using software IDE Arduino Uno. Measurements were made by comparing the measurement result using the system design and amperemeter. The second comparison measurement results get the determinant value reached 0.9976. Clamp-meter sensitivity can be seen from the results of measurements carried out repeatedly. Here researchers do ten repetitions of the measurement Clamp-meter, with the value of the determinant 0.997. It also obtained an average accuracy of the system 0,96 with a standard deviation of 0.21 , and percent error reaches $0.08 \%$. Thus concluded that the design Clamp-meter has a high enough accuracy.

\section{REFERENCES}

[1] Y. Yulkifli, Z. Afandi and Yohandri, "Development of Gravity Acceleration Measurement using Simple Harmonic Motion Pendulum Method Based on Digital tecnologi and Photogate Sensor," IOP Conference Series: Materials Science and Engineering, 2018.

[2] Flux, Clamp meter ABCs, Corporation USA, 2010.

[3] D. Mitra et al., "GMR Magnetic Sensor, Technology and Application Development," Proceedings of Scientific Meeting XXV HFI Central Java and Yogyakarta, 2011.

[4] Datasheet, “Application Notes for GMR Sensors," NVE Corporation, Book Catalog.

[5] Datasheet, “AD620,” www.analog.com, 1999.

[6] M. Iqbal, Y. Yulkifli and Y.Darvina, "Construction of Measuring System Using Temperature And Humidity Sensor SHT75 Based Internet Of Things With smarthphone Display," Periodic Physics, 2019, vol 22. no. 3.

[7] Z. Budiarso et al., "Implementation Techniques I / O Interfacing Arduino-based," DYNAMIC Information Technology Journal, Jan. 2015, vol. 20, no. 1.

[8] H. Guntoro et al., "Design of Magnetic Door Lock Using Keypad And Solenoid Based Microcontroller Arduino Uno," ElecTrans Journal, 2013, vol. 12, no. 1.

[9] Y. Yulkifli et al., "Development of color detector using colorimetry system with photodiode sensors for determination of food dye application," IOP Conference Series: Journal of Physics: Conf Series 118012031, 2019.

[10] Datasheet, "LCD," www.Alldatasheet.com, 2009. 